\title{
Bridging Atomistic/Continuum Scales in Solids with Moving Dislocations
}

\author{
TANG Shao-Qiang(唐少强) ${ }^{1 * *}$, LIU Wing K. ${ }^{2 * * *}$, KARPOV Eduard G. ${ }^{3}$, HOU Thomas Y.(侯一钊 $)^{4}$ \\ ${ }^{1}$ LTCS, Department of Mechanics and Aerospace Engineering, Peking University, Beijing 100871 \\ ${ }^{2}$ Department of Mechanical Engineering, Northwestern University, Evanston, IL 60208, USA \\ ${ }^{3}$ Department of Mechanical Engineering, Northwestern University, Evanston, IL 60208, USA \\ ${ }^{4}$ Applied and Computational Mathematics, California Institute of Technology, Pasadena, CA 91125, USA
}

(Received 19 June 2006)

\begin{abstract}
We propose a multiscale method for simulating solids with moving dislocations. Away from atomistic subdomains where the atomistic dynamics are fully resolved, a dislocation is represented by a localized jump profile, superposed on a defect-free field. We assign a thin relay zone around an atomistic subdomain to detect the dislocation profile and its propagation speed at a selected relay time. The detection technique utilizes a lattice time history integral treatment. After the relay, an atomistic computation is performed only for the defect-free field. The method allows one to effectively absorb the fine scale fluctuations and the dynamic dislocations at the interface between the atomistic and continuum domains. In the surrounding region, a coarse grid computation is adequate.
\end{abstract}

PACS: $61.72 . \mathrm{Bb}, 02.70 . \mathrm{Ns}, 61.50 . \mathrm{Ah}$

Simulating solids with moving dislocations remains one of the challenges in materials science. At a continuum level, a number of theories have been developed for different applications. These theories are based on phenomenological descriptions of dislocations, which are usually considered as continuously distributed. ${ }^{[1,2]}$ On the other hand, growing computing power allows us to explore deeper into many important features that heavily depend on the atomistic length scale, such as dislocation nucleation, mobility, crack propagation, and nano-indentation. ${ }^{[3-7]}$ To permit cost-effective simulations with a concurrent multiscale method, we perform atomistic computation only in a small subdomain, and adopt a continuum description in the surrounding region. However, the connection of these two descriptions in different scales can be delicate. If they are directly coupled, reflections usually appear at the interface, and eventually pollute the accuracy in the atomistic modelling. ${ }^{[8]}$ A proper treatment should be devised on the interface. In this Letter, we propose a numerical method to bridge the atomistic/continuum descriptions with a relay zone technique.

It should be noticed that there are various interfacial treatments for simulations with defect-free interfaces, ${ }^{[9-12]}$ as well as a few static or quasi-static treatments for dislocations. ${ }^{[13-15]}$ A dynamic treatment is devised in the present method.

The method consists of three parts: a domain decomposition, a displacement decomposition, and a time integration scheme. It can be applied to multiple dislocations in the three space dimensions, provided that there is a continuum theory compatible with the atomistic dynamics. Here we present numerical illus- trations in a $1 \mathrm{D}$ model with a single dislocation.

Consider a lattice of atoms in domain $\Omega$ governed by Newton's second law

$$
M \ddot{u}=-\nabla_{u} U(u),
$$

where $M$ is the diagonal mass matrix, $u=$ $\left(u_{1}, \cdots, u_{n_{A}}\right)^{T}$ the displacement vector with $u_{n}(t)$ the displacement at the $n$-th atom, and $U(u)$ the interatomic potential.

For the sake of clarity, we describe a method for a single dislocation. The domain $\Omega$ is separated into three subdomains, including an atomistic domain $\Omega_{D}$, a relay zone $\Omega_{R}$, and a pure continuum domain $\Omega_{P}$. By construction, strongly nonlinear wave interactions at the atomistic length scale occur only in $\Omega_{D}$. A coarse grid description is adequate to describe the dynamics away from there, except for a superposed dislocation. In particular, atomistic dynamics are not resolved in $\Omega_{P}$. This reduces the computing load and the memory requirement efficiently. An atomistic description and a coarse scale description exist simultaneously in the relay zone $\Omega_{R}$, as shown in Fig. 1 .

A dislocation is represented in the atomistic level by a spatially localized sharp jump with a magnitude of $h_{a}$, the atomistic spacing at rest. We assume that it is well resolved by atoms within a radius on the order of $h_{a}$. If the atomistic displacement is available, the position of the dislocation $x^{d}(t)$ is characterized by the maximum strain in a discrete sense $\left(\left|u_{n+1}(t)-u_{n}(t)\right|\right.$ in $1 D)$. In contrast, the dislocation profile is generally unavailable during propagation. Let $t_{E}$ be the time when the dislocation fully enters $\Omega_{R}$, and $t_{L}$ be the maximal time when the dislocation remains inside $\Omega_{R}$. For a given $\Delta T$, we take a relay time $t_{R}=t_{E}+\Delta T$.

\footnotetext{
** To whom correspondence should be addressed. Email: maotang@pku.edu.cn

*** To whom correspondence should be addressed. Email: w-liu@northwestern.edu

(C) 2007 Chinese Physical Society and IOP Publishing Ltd
} 
If the relay zone is selected large enough, the dislocation remains in $\Omega_{R}$ at $t_{R}$, namely $t_{R}<t_{L}$. We then compute a propagation speed of the dislocation in $\Omega_{R}$ from $v=\left(x^{d}\left(t_{R}\right)-x^{d}\left(t_{E}\right)\right) /\left(t_{R}-t_{E}\right)$, and a dislocation profile $u^{d}\left(t_{R}\right)$ at the relay time in a way described in the following.

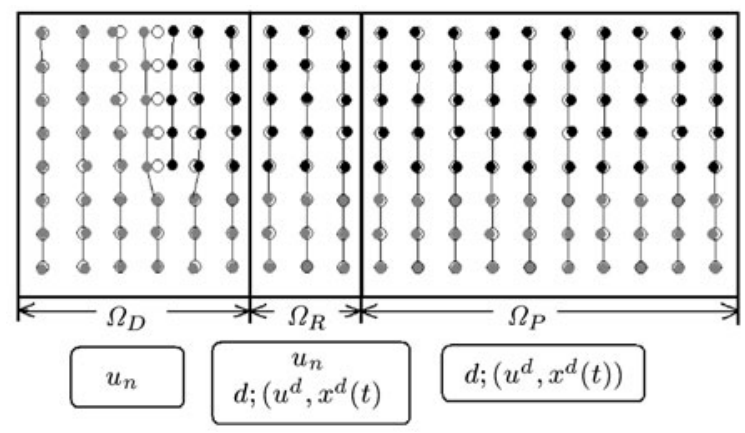

Fig. 1. Decomposition of the computing domain. Empty circles indicate the positions of the atoms at rest. Grey ones correspond to atoms that have moved for a distance of $h_{a}$ approximately. The black circles indicate those that remain close to their equilibrium positions. The boundary of domains comprised of black and grey atoms forms a dislocation core, depicted by the left-most half column of the black atoms.

We decompose the displacement field in two steps.
First, we define an abrupt dislocation profile $u^{d}(t)$ for $t<t_{R}$ as an abrupt jump, exactly of the magnitude $h_{a}$. The position of the jump is located at $x^{d}(t)$, as computed from $u$ in $\Omega_{D} \cup \Omega_{R}$. On the other hand, for $t \geq t_{R}$, we treat the dislocation propagation either by an existing continuum theory, or by maintaining the dislocation profile at $t_{R}$ and propagating at the speed $v$. As shown in Fig. 2, we decompose the displacement for all time $t>0$ by

$$
u(t)=u^{d f}(t)+u^{d}(t) .
$$

For the defect-free part of the displacement $u^{d f}(t)$, we adopt a pseudo-spectral multiscale scale method to make a second step of displacement decomposition. ${ }^{[16]}$ Taking a coarse grid with nodal points $y_{J}(J=$ $\left.1, \cdots, n_{C}\right)$ and an interpolation matrix $N$ containing $n_{C}$ normal modes for long waves, we decompose the displacement as

$$
u^{d f}=\bar{u}+u^{\prime},
$$

where a mean displacement $\bar{u}$ and a fine fluctuation part $u^{\prime}$ are obtained by linear projections.

$$
\bar{u}=N N^{T} M u^{d f}, \quad u^{\prime}=u^{d f}-N N^{T} M u^{d f} .
$$
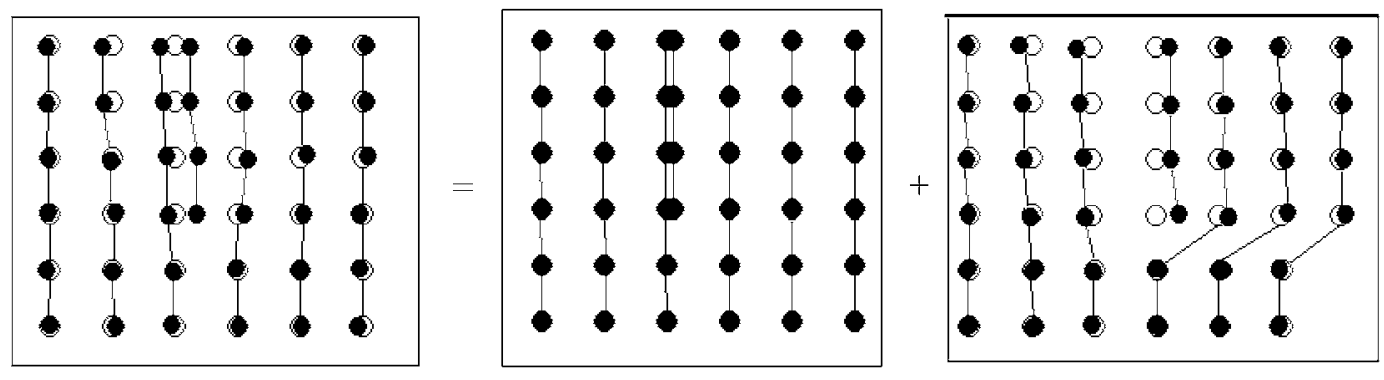

Fig. 2. Decomposition of the displacement into a dislocation part and a defect-free part.

The coarse grid displacement $d=\left(d_{1}, \cdots, d_{n_{C}}\right)^{T}$ and $\bar{u}$ have identical amplitudes for their $n_{C}$ long waves. An efficient and accurate scheme is designed for $d(t)$ through a matching differential operator approach. ${ }^{[16]}$ This gives $\bar{u}(t)$ as wanted. A reassignment of $d$ at each time step enforces consistency between the coarse grid description and the atomistic description in $\Omega_{D} \cup \Omega_{P}$.

For the fine fluctuation part, the pseudo-spectral multiscale scale method adopts a time history integral treatment to reduce the interfacial reflections for these waves. In fact, noticing that the fine fluctuation is small in amplitude, we may regard the interatomic force depending linearly on the fine fluctuation over the continuum in $\Omega_{D} \cup \Omega_{P}$. Making use of this linearity and assuming that the fine fluctuation waves propagate from $\Omega_{D}$ outward, we may reproduce all the outgoing waves from the time history of atom displacements along the interfacial layer. In particular, we have a convolution expression for the fine fluctuation in $\Omega_{R}$ as $u_{R}^{\prime}(t)=\left(\Phi * u_{I_{1}}^{\prime}\right)(t)$, where $u_{I_{1}}^{\prime}$ corresponds to the interfacial layer in $\Omega_{D}$. The time history kernel matrix $\Phi$ is essentially a lattice response function discussed by many authors, e.g. Refs. $[10,17,18]$. Similarly, we may consider the interface between $\Omega_{R}$ and $\Omega_{P}$, and express $u_{G}^{\prime}$ (the fine fluctuation for atoms in the interfacial layer in $\Omega_{P}$ side) in terms of $u_{I_{2}}^{\prime}$. More precisely, we have $u_{G}^{\prime}(t)=\left(\Theta * u_{I_{2}}^{\prime}\right)(t)$, where $\Theta$ is computed similarly to $\Phi$. It has been demonstrated that this time history integral treatment damps out fine fluctuations efficiently. ${ }^{[16]}$

We remark that the interfacial conditions are derived for defect-free problems. Noticing that the dislocation is localized, and linearly superposed in $\Omega_{R}$ and 
$\Omega_{P}$, the utilization of a defect-free multiscale method is justified in this case.

Now we are ready to describe the scheme for time integration. (1) For all time $t$, we compute $d(t)$ with the coarse scale scheme and the reassignment technique. (2) For $t \leq t_{R}$, we perform atomistic computations in $\Omega_{D} \cup \Omega_{R}$. The time history is recorded for $u_{I_{2}}^{\prime}(t)$, which provides the interfacial condition $u_{G}(t)=\bar{u}_{G}(t)+\left(\Theta * u_{I_{2}}^{\prime}\right)(t)$, with $\bar{u}_{G}(t)$ obtained through interpolation of $d(t)$. (3) The time history of $u_{I_{1}}^{\prime}(t)$ is recorded from $t_{E}$ to $t_{R}$. We compute at $t=t_{R}$, the defect-free part $u_{R}^{d f}\left(t_{R}\right)=\bar{u}_{R}\left(t_{R}\right)+$ $\left(\Phi * u_{I_{1}}^{\prime}\right)\left(t_{R}\right)$. The dislocation profile is then obtained from $u_{R}^{d}\left(t_{R}\right)=u_{R}\left(t_{R}\right)-u_{R}^{d f}\left(t_{R}\right)$. (4) For $t>t_{R}$, we consider the atomistic computation for the defect-free part $u^{d f}$. Due to the assumptions on the dislocation, we ignore the dislocation first and solve the Newton second law. The dislocation is superposed afterwards, either by a suitable continuum theory or by shifting the profile $u^{d}\left(t_{R}\right)$ at a constant speed $v$.

Now we present a numerical test for a 1D FrenkelKontorova model. ${ }^{[19]}$ In this model, the influence of atoms out of the dislocation gliding line is simplified to a periodic potential with period $h_{a}$. After rescaling, the model reads

$$
\ddot{u}_{n}=\omega^{2}\left(u_{n-1}-2 u_{n}+u_{n+1}\right)-\sin u_{n} .
$$

\section{(a)}

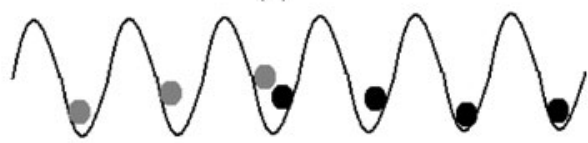

(b)

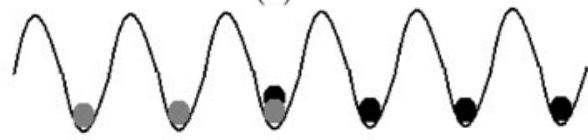

(c)

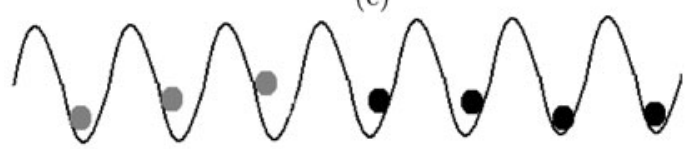

Fig. 3. Decomposition of the displacement in 1D: (a) full displacement, (b) dislocation profile, (c) defect-free part.

Here $\omega$ is the characteristic frequency. In our simulations, we take $\omega^{2}=1$. To demonstrate the reflection reduction of the proposed method, we consider the case without mean motion. The decomposition of displacement for this model is depicted in Fig. 3. Therefore, we numerically consider 512 atoms, where $\Omega_{D}$ contains the first 472 atoms, and $\Omega_{R}$ contains the next 40 atoms. A dislocation is put initially at the 451 st atom. In Fig. 4, we depict the displacement $u_{n}(t)$ for atoms near the dislocation core. It is computed with a time history technique for the boundary ${ }^{[10]}$ at finite temperature. In the cross section of $t=0$, the dislocation is represented by the smoothed step function. It moves toward the right boundary. Due to the sharp jump of the dislocation profile, a reflected dislocation forms, which eventually enters $\Omega_{D}$ and pollutes the simulation in this domain. In contrast, with the relay zone technique, we obtain from the numerical simulations a smooth passing of the dislocation into the right boundary, which would be the interface for the pure continuum subdomain $\Omega_{P}$. No reflected dislocation forms. The outgoing fine fluctuations are absorbed by the time history integral treatment. Moreover, the thermal effects may be readily included in the current formulation. For instance, with the same dislocation profile as in Fig. 4, the smooth passing of the dislocation is presented in Fig. 5. The fine fluctuations after the passing of the dislocation is mainly due to the thermal fluctuation. A more detailed analysis shows a minor change in propagation speed, compared with the zero temperature case.

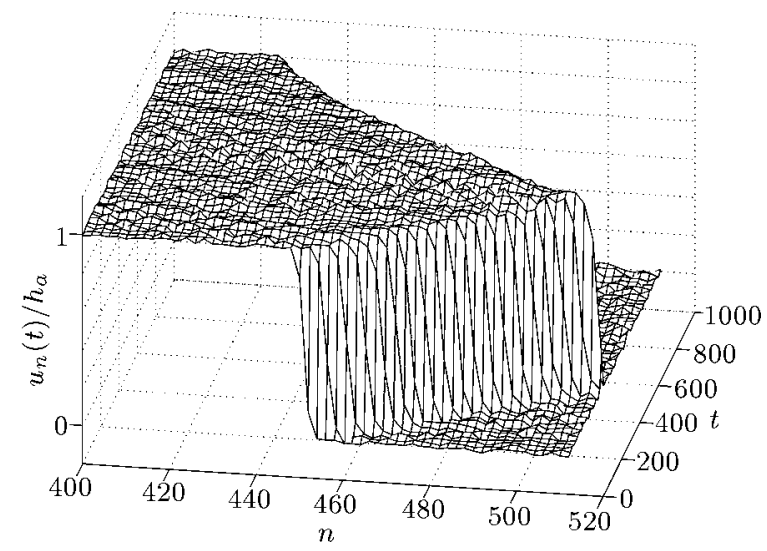

Fig. 4. Moving dislocation in $1 \mathrm{D}$ without the relay zone technique. Dislocation core is represented by the steplike displacement profile. Small fluctuations represent thermal motion, as well as waves emitted by the moving dislocation.

Finally, we make some comments. For the first thing, the abrupt profile of the dislocation before the relay does not affect the validity of the method. The atomistic computation is performed for the whole displacement that contains the dislocation. The displacement decomposition with the abrupt dislocation profile does not affect the atomistic dynamics, and has negligible influence on the coarse scale displacement due to its small length scale. The impact on the fine fluctuations matters only for the time history at the interfacial atoms, which is also negligible due to the locality of the dislocation. Secondly, in the displacement decomposition, we obtain $u^{d f}(t)$ for both $\Omega_{D}$ and $\Omega_{R}$; a coupling to the continuum theory is then straightforward.

We further remark that for multi-dimension simu- 
lations, we need to account for the topological change after the relay of dislocation. More precisely, at the moment when the dislocation propagates out of $\Omega_{R}$, the interfacial layer in $\Omega_{R}$ needs to be modified. ${ }^{[20]}$ As an example, consider a lattice shown in Figs. 1 and 2, when the half-column heavy dots (atoms at the dislocation core) shift across $\Omega_{R}$, we either delete the upper half-column, or add another half-column in the lower to form a full column of atoms. For the latter choice, the defect-free part for the added atoms is readily obtained from the convolution expression for the fine fluctuation, and the interpolation of $d$ for the mean displacement. Despite of a set of more complicate time history kernel functions, the multi-dimension simulations with dislocations may be treated in the same way as in one space dimension.

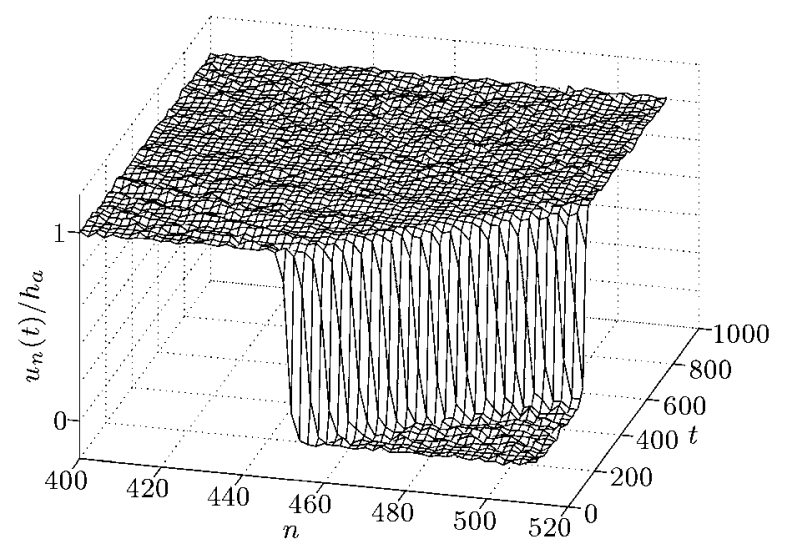

Fig. 5. Moving dislocation in 1D with the relay zone technique.

In summary, we have proposed a dynamic treatment for multiscale simulations in solids with moving dislocations. While many existing multiscale methods can be utilized to damp out efficiently the fine fluctuations of small amplitude (compared with the atomistic spacing) at an interface of different scales, passing a dislocation across such an interface usually results in strong reflections. The difficulty stems from the finite amplitude of jump in a dislocation profile, which is of the same order of magnitude as the atomistic spacing. We develop a relay zone technique and couple it with the pseudo-spectral multiscale method to remove the reflections. The key idea is to single out the disloca- tion part from the total displacement $u$, after the relay time $t_{R}$ on the basis of a time-history integral. The relay zone $\Omega_{R}$ serves for three purposes, namely, to reduce reflection for waves from $\Omega_{D}$, to detect the speed and profile of the dislocation, and to provide boundary conditions for the coarse scale computation in $\Omega_{P}$ during the reassignment of $d$. Numerical tests demonstrate the method to be effective. Moreover, the relay zone technique may be utilized together with many existing multiscale methods.

The authors would like to thank Professors S. Lichter and S. Hao for stimulating discussions. The work has been conducted under the support of NSF and ARO, and NSFC No. 90407021.

\section{References}

[1] van der Giessen E and Needleman A 1995 Model. Simul. Mater. Sci. Eng. 3689

[2] Hao S, Liu W and Weertman J 2004 Phil. Magn. 841067

[3] Bulatov V and Cai W 2002 Phys. Rev. Lett. 89115501

[4] Li J, van Vliet K, Zhu T, Yip S and Suresh S 2002 Nature 418307

[5] Landman U, Ludtke W, Burnham N and Colton R 1990 Science $\mathbf{2 4 8} 454$

[6] Rieth M and Schommers W 2005 Handbook of Theoretical and Computational Nanotechnology (Stevenson Ranch, CA: American Scientific Publishers)

[7] Liu W K, Karpov E G, Zhang S and Park H S 2004 Comp. Meth. Appl. Mech. Eng. 1931529

[8] Liu W K, Karpov E G and Park H S 2005 Nano Mechanics and Materials: Theory, Multiscale Methods and Applications (New York: Wiley)

[9] Abraham F et al 1998 Comput. Phys. 12538

[10] Cai W, de Koning M, Bulatov V and Yip S 2000 Phys. Rev. Lett. 853213

[11] To A and Li S 2005 Phys. Rev. B 72035414

[12] Wagner G J and Liu W K 2003 J. Comput. Phys. 190249

[13] Tadmor E, Ortiz M and Phillips R 1996 Philos. Magn. A 731529

[14] Tadmor E, Miller R, Phillips R and Ortiz M 1999 J. Mater. Res. 142233

[15] Shilkrot L, Miller R and Curtin W 2002 Phys. Rev. Lett. 89025501

[16] Tang S, Hou T Y and Liu W K 2006 J. Comput. Phys. 21357

[17] Wagner G J, Karpov E G and Liu W K 2004 Comp. Meth. Appl. Mech. Eng. 1931579

[18] Karpov E G, Wagner G J and Liu W K 2005 Int. J. Numerical Meth. Eng. 621250

[19] Braun O and Kivshar Y 1998 Phys. Rep. 3061

[20] Shilkrot L E, Curtin W A and Miller R E 2002 J. Mech. Phys. Solids 502085 